\title{
Acute gastric ulcer associated with gamma knife treatment (conformal stereotactic radiotherapy) of recurrent hepatocellular carcinoma
}

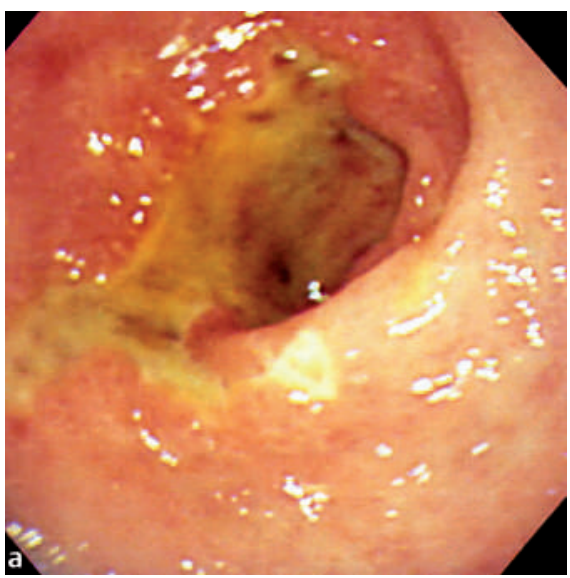

E154

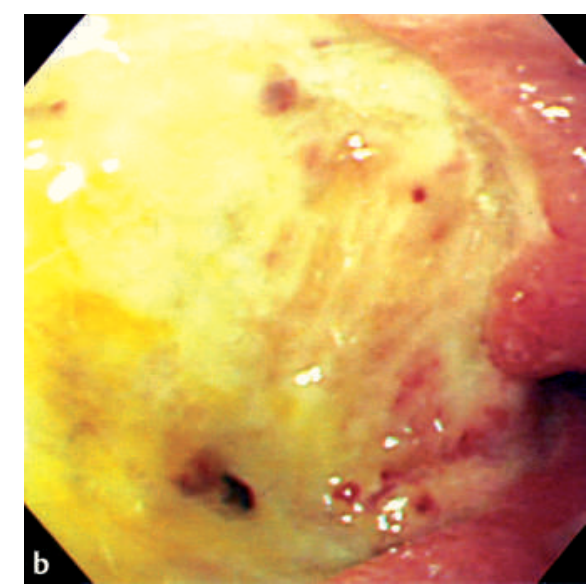

Figure 1 A 73-year-old man received one course of gamma knife radiotherapy (conformal stereotactic radiotherapy) for recurrent hepatocellular carcinoma, with a total dose of $45 \mathrm{~Gy}$ given within 1 month. Three days after finishing radiotherapy, he developed tarry stools. At esophagogastroduodenoscopy, he was found to have a large gastric ulcer on the lesser curvature and the anterior wall of the antrum, which had an irregular shape and margins (a) and several exposed vessels (b).

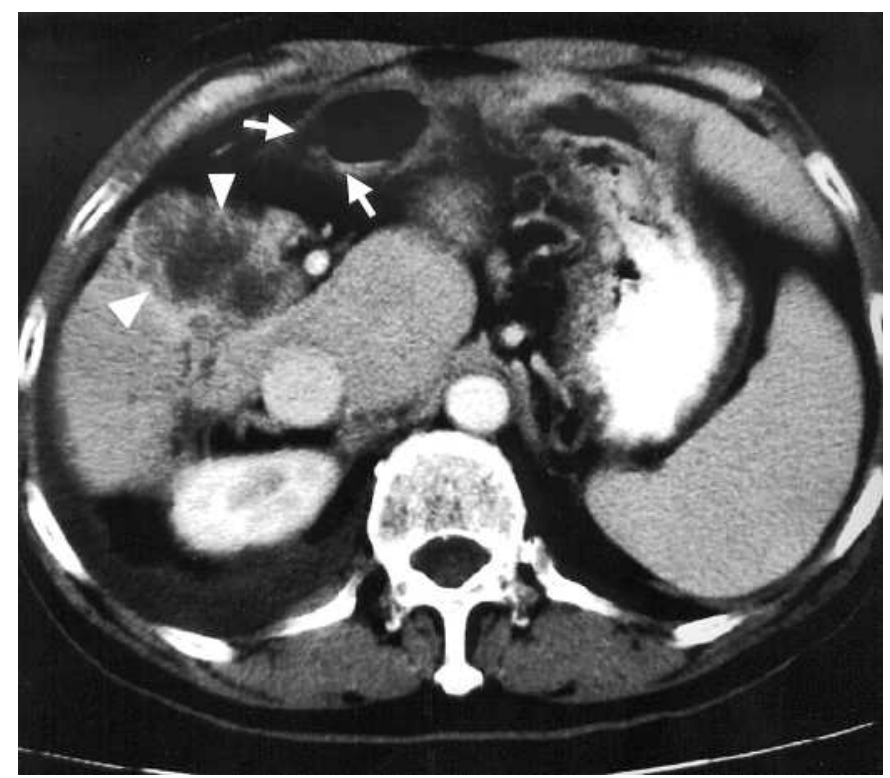

Figure 2 Abdominal computed tomography showed that there was a close anatomic relationship between the gastric antrum (arrows) and the hepatocellular carcinoma in the residual left hepatic lobe (arrowheads).

C. Y. Chang', C. H. Tu' ${ }^{1}$, T. S. Chiang', H. P. Wang ${ }^{2}$

${ }^{1}$ Department of Internal Medicine, E-DA Hospital, Kaohsiung, Taiwan

2 Department of Emergency Medicine, National Taiwan University Hospital and National Taiwan University College of Medicine, Taipei, Taiwan

Endoscopy_UCTN_Code_CCL_1AB_2AD_3AC

\section{Corresponding Author}

\section{H. P. Wang M.D.}

Department of Emergency Medicine National Taiwan University Hospital No. 7 Chung-Shan South Road Taipei

Taiwan

Fax: $\quad+886-2-23947899$

E-mail: whp@ha.mc.ntu.edu.tw 